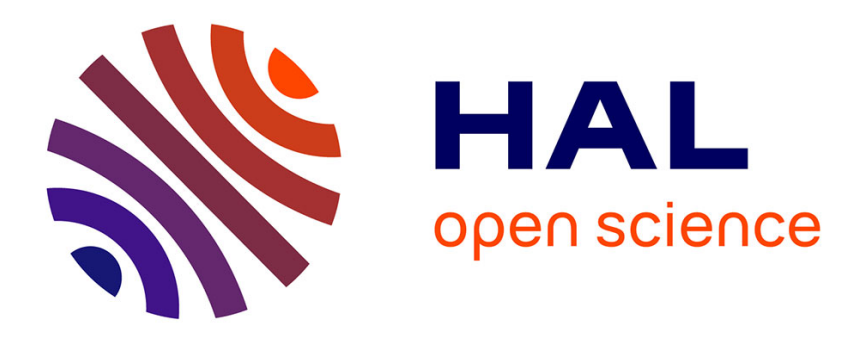

\title{
Nuclear relaxation and spin dynamics in NMP TCNQ
} F. Devreux, M. Guglielmi, M. Nechtschein

\section{To cite this version:}

F. Devreux, M. Guglielmi, M. Nechtschein. Nuclear relaxation and spin dynamics in NMP TCNQ. Journal de Physique, 1978, 39 (5), pp.541-547. 10.1051/jphys:01978003905054100 . jpa-00208785

\section{HAL Id: jpa-00208785 https://hal.science/jpa-00208785}

Submitted on 1 Jan 1978

HAL is a multi-disciplinary open access archive for the deposit and dissemination of scientific research documents, whether they are published or not. The documents may come from teaching and research institutions in France or abroad, or from public or private research centers.
L'archive ouverte pluridisciplinaire HAL, est destinée au dépôt et à la diffusion de documents scientifiques de niveau recherche, publiés ou non, émanant des établissements d'enseignement et de recherche français ou étrangers, des laboratoires publics ou privés. 


\author{
Classification \\ Physics Abstracts \\ $76.60-71.45 \mathrm{G}-71.15 \mathrm{~N}$
}

\title{
NUCLEAR RELAXATION AND SPIN DYNAMICS IN NMP TCNQ
}

\author{
F. DEVREUX $(*)$, M. GUGLIELMI and M. NECHTSCHEIN $\left({ }^{*}\right)$ \\ Institut de Recherche Fondamentale, Département de Recherche Fondamentale, \\ Section de Résonance Magnétique, Centre d'Etudes Nucléaires de Grenoble, \\ 85 X 38041 Grenoble Cedex, France
}

(Reçu le 2 décembre 1977, accepté le 2 février 1978)

\begin{abstract}
Résumé. - Des mesures de relaxation nucléaire des protons ont été effectuées en fonction de la fréquence dans des échantillons de NMP TCNQ sélectivement deutériés. Les résultats montrent que la principale source de relaxation nucléaire provient de spins électroniques localisés sur les chaînes de NMP et associés à un transfert de charge incomplet. Après soustraction de leur contribution à la relaxation, il apparaît que la dynamique de spin dans les chaînes de TCNQ a un caractère diffusif unidimensionnel, comme dans les autres sels conducteurs de TCNQ.
\end{abstract}

\begin{abstract}
Proton nuclear relaxation measurements have been performed as a function of the frequency in selectively deuterated NMP TCNQ compounds. The results show that the main source of nuclear relaxation is provided by localized electronic spins, associated with back charge transfer on the NMP stacks. Once their contribution to the relaxation has been subtracted, it appears that the spin dynamics in the TCNQ stacks has a one-dimensional diffusive behaviour, as in other TCNQ conducting compounds.
\end{abstract}

1. Introduction. - As a first approximation, the conducting salts of tetracyanoquinodimethane (TCNQ) can be divided into two classes [1]. On one hand, the tetrathiofulvalene TCNQ (TTF TCNQ) family with incomplete charge transfer and two conducting chains presents a sharp three-dimensional Peierls ordering. On the other hand, the compounds with diamagnetic and asymmetric cations display a smooth one-dimensional (1D) change from a high temperature metallic regime to low temperature semiconducting behaviour with disorder induced localized states.

As far as the transport properties $[2,3]$ are concerned, N-methyl Phenazinium TCNQ (NMP TCNQ) undoubtedly belongs to the second class. But its magnetic properties appear to present rather peculiar features. The susceptibility $(\chi)$ actually begins to increase with decreasing temperature for temperature less than $200 \mathrm{~K}$ [3], which is opposite to the behaviour of the other salts of this classlike Quinolinium $\mathrm{TCNQ}_{2}\left(\mathrm{Qn} \mathrm{TCNQ}_{2}\right)$ - which show flat or slightly decreasing susceptibility down to $20 \mathrm{~K}$ [1]. Moreover the nuclear relaxation rate $\left(T_{1}^{-1}\right)$ is strongly

(*) Centre National de Recherche Scientifique. enhanced [4] : it is one order of magnitude larger than in $\mathrm{Qn} \mathrm{TCNQ}_{2}$ [5] or TTF TCNQ [6, 7]. These features were first believed to be an effect of the $1: 1$ stoichiometry NMP TCNQ. Due to the presence of one electron per TCNQ site (instead of 0.5 for $\mathrm{Qn} \mathrm{TCNQ}_{2}$ or 0.58 for TTF TCNQ), the role of the coulombic on-site interaction can be thought to be more drastic in driving the Mott-Hubbard transition [3] and giving rise to strongly enhanced static $(\chi)$ and dynamic $\left(T_{1}^{-1}\right)$ susceptibility [4]. More recently it was claimed from optical studies [8], CW NMR measurements [9], thermoelectric power experiments (TEP) [10], comparative structural considerations [11] and X-ray diffuse scattering [12] that the charge transfer from NMP to TCNQ is incomplete.

2. Experimental. - In this work we give a direct and definitive proof of the incomplete charge transfer in NMP-TCNQ. Using a method successfully employed to determine the local spin susceptibility in TTF-TCNQ [6], we have measured the proton relaxation rates in two selectively deuterated compounds : (NMP)* TCNQ and NM*P-TCNQ. In (NMP)* TCNQ the whole cation is deuterated. In both compounds the methyl group have been 
deuterated in order to inhibit a possible relaxation mechanism via the methyl group rotation [13].

The compounds were prepared following Melby's method [14] in a glove box under an Argon atmosphere. Commercial TCNQ was used after several recrystallizations from acetonitrile. Phenazine was deuterated by two catalytic exchanges with $\mathrm{D}_{2} \mathrm{O}$ [15]. The methyl sulfate of methyl phenazinium (NMP)* M* $\mathrm{SO}_{4}$ was obtained by the action of deuterated dimethyl sulfate on previously deuterated phenazine in dry nitrobenzene. The total deuteration of (NMP)* as determined by mass spectroscopy was $98 \%$. Almost all the remaining protons were located on the phenazinium rings and corresponded to $16 \%$ of NM*P $\left(\mathrm{HD}_{7}\right)$ molecules. The complexes (NMP)* TCNQ and NM*P TCNQ were obtained from reaction of saturated cation and TCNQ solutions in acetonitrile. After cooling from boiling temperature down to room temperature, the polycristalline samples were collected, filtered and put in vacuum-sealed tubes. This procedure is expected to lead to the disordered form of NMP TCNQ.

The nuclear relaxation rates $T_{1}^{-1}$ measured at room temperature as a function of the frequency are given in figure 1 . The striking feature is that $T_{1}^{-1}$ is nearly five times larger in $\mathrm{NM}^{*} \mathrm{P}$ TCNQ than in (NMP)* TCNQ. This clearly demonstrates that the NMP protons relax much more rapidly than the TCNQ protons, and that consequently, the main relaxation mechanism lies in the NMP stacks. Since the methyl rotation mechanism is excluded by deuteration, this is direct evidence for the presence of

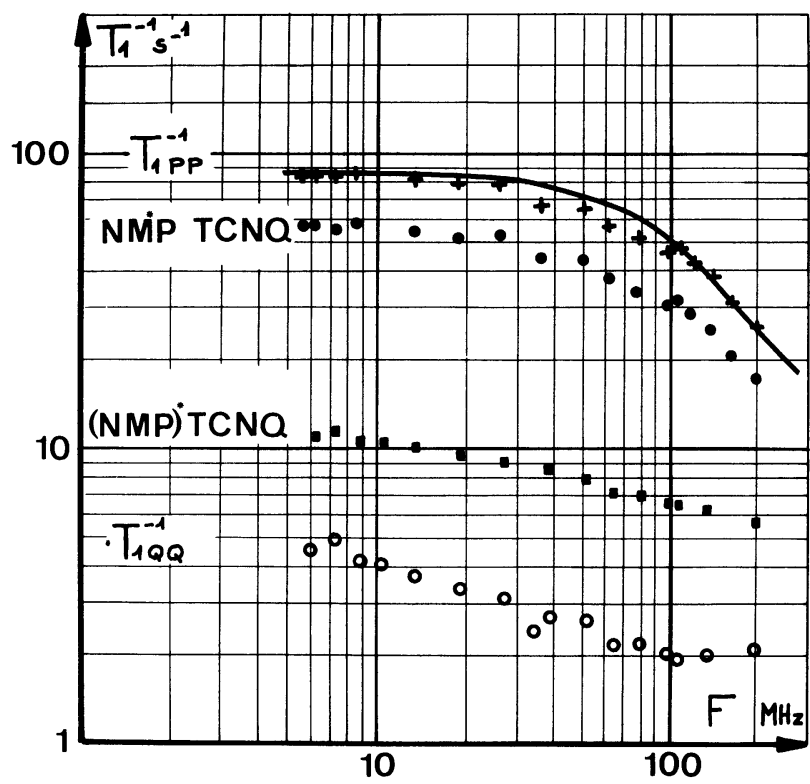

Fig. 1. - Relaxation rates as a function of the nuclear Larmor frequency $\mathrm{F}$ : (๑) measured in NM*P TCNQ, ( $\square$ ) measured in (NMP)* TCNQ, (O) intrinsic TCNQ relaxation rate as deduced from eqs. (3.1), (5.1) and (5.3) with NMP spin density model $(b)$, $(+)$ intrinsic NMP relaxation rate as deduced from eq. (3.4). The solid curve is obtained from the fit of the experimental data with a Lorentzian lineshape for the frequency dependent spin correlation function. electronic spins remaining on the NMP stacks, and thus for incomplete charge transfer.

It is noteworthy that, contrary to the case of (NMP)* TCNQ the relaxation in NM*P TCNQ is strongly non-exponential. (The data given in figure 1 have been obtained by considering the recovery of the magnetization at time between $t=0$ and $t=T_{1}$.) Furthermore it appears that the NMR line is inhomogeneous. A detailed study shows that the protons which have the shortest $T_{2}$ also have the shortest $T_{1}$. This is evidence that the internal magnetic field is inhomogeneous and that the electronic spins of the NMP stacks are localized. The presence of localized electronic spins on the NMP sites has already been noticed by Butler, Wudl and Soos [9] from observation of the field dependence of the NMR second moment. We here establish that these localized spins are the main source for the nuclear relaxation in NMP TCNQ. This clears up the problem of the enhanced nuclear relaxation rate in NMP TCNQ [4]. This also shows that the spin dynamics of the TCNQ chain in NMP TCNQ cannot be studied from $T_{1}$ measurements, unless these measurements are performed on cation deuterated compounds. Even in a cation deuterated compound, the contribution of the NMP electronic spins to the relaxation of the TCNQ protons should be taken into account and subtracted in order to obtain the intrinsic TCNQ relaxation.

3. Analysis of the results. - Denoting TCNQ and NMP by the subscripts $Q$ and $P$ respectively we write the relaxation rate of the TCNQ protons (measured in (NMP)* TCNQ) as :

$$
T_{1 \mathrm{Q}}^{-1}=T_{1 \mathrm{QQ}}^{-1}+T_{1 \mathrm{PQ}}^{-1}
$$

where we have distinguished the intrinsic TCNQ contribution (QQ) from that of the NMP spins (PQ). In a similar way, one has for the phenazinium protons :

$$
T_{1 \mathrm{P}}^{-1}=T_{1 \mathrm{PP}}^{-1}+T_{1 \mathrm{QP}}^{-1} .
$$

The relaxation rate for the mixed compound $\mathrm{NM}^{*} \mathrm{P}$ TCNQ is expressed as :

$$
T_{1}^{-1}=\frac{1}{3} T_{1 \mathrm{Q}}^{-1}+\frac{2}{3} T_{1 \mathrm{P}}^{-1}
$$

where the coefficients $\frac{1}{3}$ and $\frac{2}{3}$ account for the relative number of protons in TCNQ and in $\mathrm{NM}^{*} \mathrm{P}$, respectively.

Since it turns out from the measured values that $\frac{1}{3} T_{1 \mathrm{Q}}^{-1} \ll T_{1}^{-1}$ in the whole frequency range, one can neglect the first term on the right hand side of eq. (3.3). Furthermore the TCNQ contribution to the relaxation of the phenazinium protons is also negligible compared to the intra NMP process. Therefore one has :

$$
T_{1 \mathrm{PP}}^{-1} \simeq T_{1 \mathrm{P}}^{-1} \simeq \frac{3}{2} T_{1}^{-1}
$$


Thus the measurements of $T_{1}$ in NM*P TCNQ and indeed in NMP TCNQ are a direct probe of the spin dynamics on the NMP stacks.

4. NMP spin dynamics. - The frequency dependence of the intrinsic relaxation rate on the NMP stacks $\left(T_{1 \mathrm{PP}}^{-1}\right)$, deduced from eq. (3.4), is given in figure 1 .

An analysis of this result provides further information on the back charge transfer and, more generally, on the NMP spin dynamics. The fact that the charge transfer is not complete in NMP TCNQ differentiates this compound from Qn $\mathrm{TCNQ}_{2}$, for which the occurrence of a complete charge transfer has been established from nuclear relaxation measurements [16]. Clearly a complete charge transfer would involve weaker coulombic repulsion between the conduction electrons in a $1: 2$ salt-such as $\mathrm{Qn} \mathrm{TCNQ}_{2}-$ than in NMP TCNQ. It is noteworthy that in TTF TCNQ, which has the same stoichiometry as NMP TCNQ, the charge transfer is 0.58 . However, in contrast to what occurs in TTF, the electronic spins in the NMP stacks are localized over one or a few sites. This is shown by the inhomogeneous character of the NMP proton relaxation, as pointed out before. More quantitatively an upper bound can be obtained for the rate of motion of the NMP spins. Assuming a random walk motion with a jump frequency $v_{j}$, the number of NMP sites which are visited by a given electronic spin during a period of time $\tau$ is $\left(v_{j} \tau\right)^{1 / 2}$. The observation of an inhomogeneous relaxation implies that all the sites are not visited during a time $\tau \simeq T_{1 \mathrm{PP}}$, that is :

$$
C\left(v_{j} T_{1 \mathrm{PP}}\right)^{1 / 2}<1
$$

where $C$ is the NMP electronic spin concentration.

Taking for $C$ the value estimated below $\left(C \sim 10^{-1}\right)$ one obtains : $v_{j}<10^{2}-10^{3} \mathrm{~s}^{-1}$ which means quasistatic spins on the NMP stacks. It is noteworthy that in the case of TCNQ chains, the spins jump rapidly from site to site $\left(v_{j}>10^{10} \mathrm{~s}^{-1}\right)$. For instance in the semi-conducting salt $\varphi_{3} \mathrm{AsCH}_{3} \mathrm{TCNQ}_{2}$ the recovery of the nuclear magnetization remains exponential even with exciton concentration as low as $0.1 \%$ [17].

The amount of NMP electronic spins can be estimated from the $T_{1}$ data. Neglecting a possible nuclear spin diffusion effect, the relaxation rate in the NMP stacks is expressed as [18] :

$$
\begin{aligned}
T_{1 \mathrm{PP}}^{-1}=C \frac{\pi}{10}[ & 3\left\langle d_{\mathrm{P}}^{2}\right\rangle f_{\mathrm{P}}\left(\omega_{\mathrm{N}}\right)+ \\
& \left.+\left(7\left\langle d_{\mathrm{P}}^{2}\right\rangle+5\left\langle a_{\mathrm{P}}^{2}\right\rangle\right) f_{\mathrm{P}}\left(\omega_{\mathrm{e}}\right)\right]
\end{aligned}
$$

where $\left\langle d_{\mathrm{P}}^{2}\right\rangle$ and $\left\langle a_{\mathrm{P}}^{2}\right\rangle$ are the mean square dipolar and scalar hyperfine couplings in the NMP stacks, $f_{\mathrm{P}}(\omega)$ is the normalized frequency correlation function of the NMP spin fluctuations, and $\omega_{\mathrm{N}}$ and $\omega_{\mathrm{e}}$ are the nuclear and electronic Larmor frequencies. In order to obtain $C$ from Eq. (4.1) and experimental data, we have to calculate the hyperfine couplings and estimate the frequency correlation function.

We assume a lorentzian line shape for $f_{\mathrm{P}}(\omega)$ :

$$
f_{\mathrm{P}}(\omega)=\frac{1}{\pi} \frac{\Delta}{\Delta^{2}+\omega^{2}}
$$

Moreover we suppose that the frequency variation observed in figure 1 arises from the $\omega_{\mathrm{e}}$ contribution and therefore we have :

$$
f_{\mathrm{P}}\left(\omega_{\mathrm{N}}\right)=f_{\mathrm{P}}(\omega=0)=(\pi \Delta)^{-1}
$$

(the alternative possibility leads to inconsistent conclusions).

As concerns the couplings, we assume a dipolar to scalar ratio : $\xi=\left\langle d_{\mathrm{p}}^{2}\right\rangle \mid\left\langle a_{\mathrm{p}}^{2}\right\rangle=1 / 5$. This result has been obtained in the case of TCNQ [19] but it should be reliable also in the case of the NMP radical because it mainly involves the aromatic character of the $\mathrm{C}-\mathrm{H}$ bond, which is common for both molecules.

With these hypotheses, eq. (4.1) becomes :

$$
\begin{aligned}
& T_{1 \mathrm{PP}}^{-1}=\frac{\pi}{2} C\left\langle a_{\mathrm{P}}^{2}\right\rangle {\left[\frac{3 \xi}{5} f_{\mathrm{P}}(\omega=0)+\right.} \\
&\left.+\left(1+\frac{7 \xi}{5}\right) f_{\mathrm{P}}\left(\omega_{\mathrm{e}}\right)\right]
\end{aligned}
$$

A fit of the experimental data with eqs. (4.2) and (4.3) has been performed in figure 1 . The lorentzian shape gives a satisfactory agreement. The spectral density line width obtained from this fit is :

$$
\Delta=4.3 \times 10^{11} \mathrm{rad} / \mathrm{s} \text {. }
$$

This value appears rather large for an impurity spectrum. What are the possible origins of this spectrum width ?

As shown above, the jump frequency is much smaller than $\Delta$. We can also discard the spin-spin couplings (dipolar and exchange) between the NMP spins which are too weak, according to the dilute character of the NMP spin system $(C \sim 0.1$ as determinated below). Thus let us turn to the interaction between NMP localized spins and TCNQ itinerant spins. Two mechanisms may contribute to the linewidth : i) hopping from NMP site to the neighbouring TCNQ chain and ii) exchange between NMP and TCNQ spins. We can estimate the values of two interchain coupling parameters (the transfer integral $t_{\perp}$ in case i) and the exchange integral $J_{\perp}$ in case ii)) which would lead to the observed $\Delta$.

The interchain hopping gives rise to a broadening of the NMP spin spectrum. However this contribution is narrowed by the electronic motion on the TCNQ stacks. The resulting line width can be evaluated from the Golden Rule [7] :

$$
\Delta \sim \frac{2 \pi}{\hbar^{2}} t_{\perp}^{2} \tau_{v}
$$


where $\tau_{v}$ is the collision time for the carriers on the TCNQ chain. Using the Drude formula, an evaluation of the $\tau_{v}$ can be obtained from the d.c. conductivity : $\tau_{v} \sim 10^{-14} \mathrm{~s}$. Eg. (4.4) thus yields $: t_{\perp} \sim 1.5 \mathrm{meV}$, the order of magnitude of which agrees with the values calculated by MO methods in TTF TCNQ [20].

Let us now consider the interchain exchange. Since the overlap between the molecular orbitals of NMP and TCNQ is very small because of the directional character of the $\pi$-orbitals, the exchange coupling between NMP and TCNQ spins results from virtual hopping involving the inter-chain transfer integral $t_{\perp}$. The interchain exchange coupling is then given by : $J_{\perp} \sim t_{\perp}^{2} / \Delta E$, where $\Delta E$ is the caracteristic energy for double occupancy of the first unoccupied orbital of $\mathrm{NMP}^{+}$. Taking into account the motional narrowing of the exchange, the line width $\Delta$ is then expressed as :

$$
\Delta \sim \frac{2 \pi}{\hbar^{2}} J_{\perp}^{2} F_{\mathrm{Q}}(\omega=0)
$$

where $F_{\mathrm{Q}}(\omega=0)$ is the zero frequency spin correlation function in the TCNQ chain. Indeed the exchange coupling is expected to be weaker than the transfer integral, but the motional narrowing is less effective, because of the 1D character of the spin diffusion [21] in the TCNQ chain (see $\S 4)$; such a $1 \mathrm{D}$ motion yields :

$$
F_{\mathbf{Q}}(\omega)=\frac{k T \tilde{\chi}_{\mathbf{Q}}}{\sqrt{2 \tilde{D} \omega}}
$$

In eq. (4.6), $\tilde{\chi}_{Q_{\tilde{\alpha}}}$ is the reduced susceptibility of the TCNQ chain $: \tilde{\chi}_{\mathrm{Q}}=\chi_{\mathrm{Q}} /\left(2 N \mu_{\mathrm{B}}^{2}\right)(N$ is the Avogadro number, $\mu_{\mathrm{B}}$ the Bohr magneton). The diffusion coefficient $D$ for the spin excitations in the TCNQ chain is related to $\tilde{D}$ by : $D=\tilde{D} a^{2}$, where $a$ is the intersite distance. In order to obtain $F_{\mathrm{Q}}(\omega=0)$ from eq. (4.6), we put : $\omega=\omega_{\mathrm{c}}$, where $\omega_{\mathrm{c}}$ is a cut-off frequency (presumably due to TCNQ-TCNQ interchain hopping). The absence of cut-off in the experimental frequency range (see $\S 4$ and Fig. 4) does not allow us to determine $F_{\mathrm{Q}}(\omega=0)$, but we can obtain a lower limit and therefore an upper limit for $J_{\perp}$ to explain the reported value of $\Delta$; it comes out as $J_{\perp} / k<12 \mathrm{~K}$.

It is difficult to ascertain which one of these two processes is dominant, but it may be seen from the above estimates that the NMP-TCNQ interactions can account for the line width of the NMP spin correlation function.

Information about the NMP spin concentration is given by the comparison of experimental data with expressions (4.2) and (4.3). It is obtained from the value of the relaxation rate at low frequency (where $\left.f_{\mathrm{P}}\left(\omega_{\mathrm{e}}\right)=f_{\mathrm{P}}\left(\omega_{\mathrm{N}}\right)=f_{\mathrm{P}}(0)\right)$ :

$$
\left.T_{1 \mathrm{PP}}^{-1}\right)_{\omega \rightarrow 0}=C\left\langle a_{\mathrm{P}}^{2}\right\rangle(1+2 \xi) / 2 \Delta .
$$

Thus an estimate of $C$ from eq. (4.7) requires a calculation of the scalar hyperfine coupling. The simplest model consists of supposing the NMP spin to be localized on one NMP molecule. In this case, $\left\langle a_{\mathrm{P}}^{2}\right\rangle$ depends only on the spin density distribution over the NMP atoms :

$$
\left\langle a_{\mathrm{P} .}^{2}\right\rangle=1 / 8 \sum_{i=1}^{8} a_{\mathrm{P} i}^{2}
$$

where the scalar coupling $a_{\mathrm{P} i}$ of a proton $\mathrm{H}_{i}$ in a given $=\mathrm{C}^{i}-\mathrm{H}_{i}$ bond is taken as : $a_{\mathrm{P} i}=\gamma_{\mathrm{e}} \sigma_{i} Q$, with $Q=-23.7 \mathrm{G}$ [22] $\gamma_{\mathrm{e}}$ is the electronic gyromagnetic factor and $\sigma_{i}$ is the spin density at carbon $\mathrm{C}_{i}$.

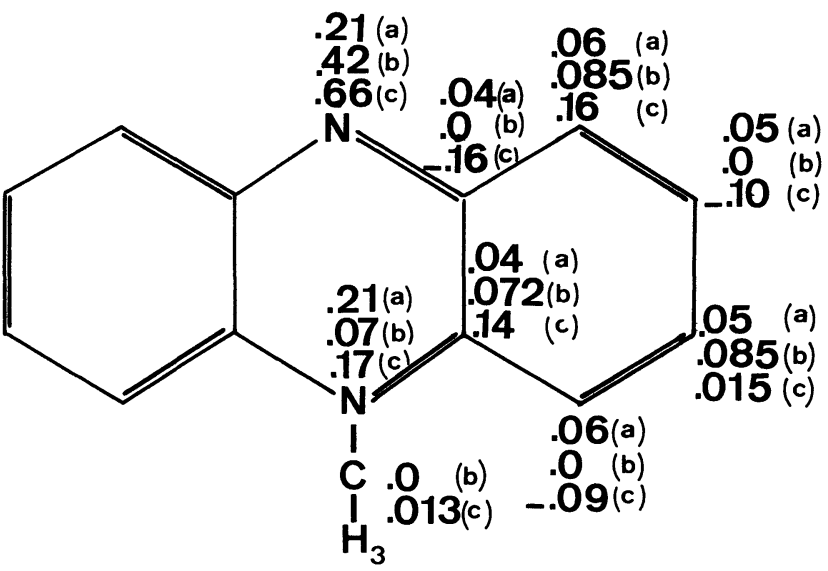

FIG. 2. - Spin density distribution in the NMP radical : (a) following Butler et al. [9], (b) and (c) obtained from Hückel-like and INDO MO calculations respectively.

In figure 2, we give three sets of spin density distribution in the NMP radical : (a) from data of ref. [9] (b) from a Huckel-like MO calculation, and (c) from an INDO MO calculation. We note large discrepancies between the different sets of data. It is difficult to ascertain which set is the best since there exists no experimental determination of the hyperfine couplings for the NMP radical. The authors of reference [9] invoke the similitarity of the NMP radical to the dihydrophenazine positive ion, for which the EPR spectrum in solution has been obtained [23]. However, the dihydrophenazine has a symmetric structure with two pyrrole type nitrogens, while the NMP radical has a pyridine type nitrogen on one side. This difference may lead to very different spin distributions, as revealed by MO calculations. It is noteworthy that INDO calculations allow negative spin densities, and, consequently, yield larger absolute values for the spin densities. However, this effect is probably over-estimated.

Using eqs. (4.7) and (4.8), the experimental values for $\Delta$ and $\left.T_{1 \mathrm{PP}}^{-1}\right)_{\omega \rightarrow 0}=86 \mathrm{~s}^{-1}$ yield for the spin concentration $C=10 \%, 8 \%$ and $2 \%$ with the spin density distributions $(a),(b)$ and $(c)$ respectively. However, it is likely that the NMP spin can be 
delocalized over more than one NMP site. We now propose a model which allows some delocalization, taking into account the fact that the electronic spins do not move along the NMP stacks. We then recalculate the effective hyperfine coupling and obtain a more realistic set of values for $C$.

In accordance with the authors of reference [9] we think that the disorder of the methyl group orientation [24] must play an essential role for the localization of the electronic spins in the NMP stacks. A schematic array of NMP sites with randomly oriented dipoles is represented in figure $3 a$ ). The

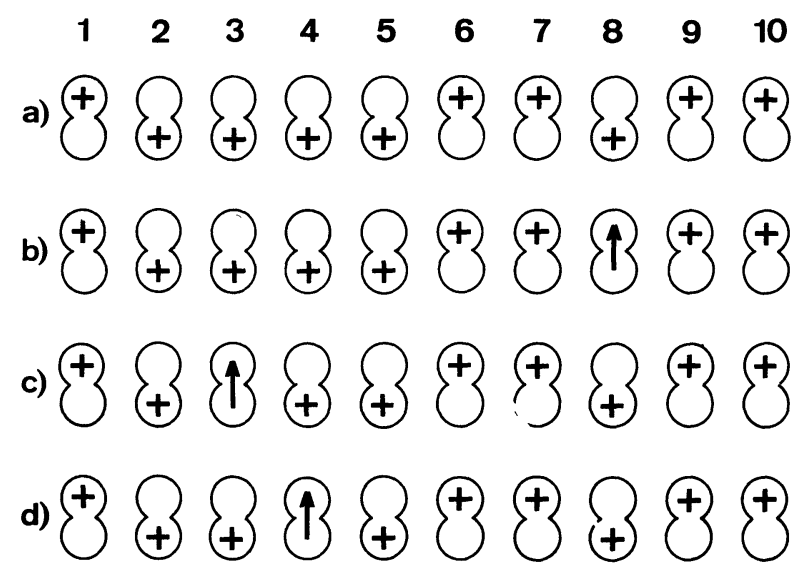

FIG. 3. - Schematic representation of a NMP stack as a disordered electric dipole chain : (a) without back charge transfer; $(b)$ with unfavourable back charge transfer; $(c)$ and $(d)$ with favourable back charge transfer (two energetically equivalent configurations).

problem of the formation of TCNQ salts with disordered cations has been discussed elsewhere [25]; here we only discuss the problem of occurrence and localization of back charge transfer. In figure $3 b$ ) we assume that there is a back charge transfer - and thus a spin - localized on site 8 . The electric dipole moment of site 8 is reduced. Consequently, the dipole-dipole attraction between this site and its nearest neighbours is lowered. Let us now assume that the back charge transfer appears on site 3 (Fig. 3c). The electric dipole moment of this site is reduced, but contrary to the previous case, this leads to a reduction of dipole-dipole repulsion with its nearest neighbours. Clearly the occurrence of a back charge transfer in the second case (Fig. $3 c$ ) is more favourable than in the first case (Fig. $3 b$ ), since it has a self-stabilizing effect.

In a general way the dipole-dipole contribution to the total energy is lowered by a back charge transfer which occurs in a cluster of NMP sites having the same dipole orientation. The back charge transfer should not be located on the boundary cluster sites. Thus the number $n$ of NMP in such a cluster should be larger than 3. If we take into account the dipole-dipole interaction between non-nearest neighbours, we realize that the larger $n$, the larger is the lowering of the dipole-dipole energy, and thus the larger is the probability for having a back charge transfer in a given cluster. However, in the following, for simplicity we neglect this effect and assume that the probability $p$ for finding an electronic spin is the same in any cluster with $n \geqslant 3$. The total NMP spin concentration is then given by :

$$
C=p \sum_{n=3}^{\infty} C(n)
$$

where $C(n)$ is the probability for having a cluster of $n$ sites. Assuming a random distribution for the dipole orientations, one has : $C(n)=n 2^{-(n+1)}$ and eq. (4.9) yields $C=0.5 p$. For $n>3$, the back charge transfer is allowed to be delocalized over more than one site. For instance, figures $3 c$ and $3 d$ represent two configurations which have the same energy. In such a situation a rapid hopping is expected between the sites 3 and 4 . Neglecting as before the dipole-dipole interaction between non-nearest neighbours, the electronic spin would be delocalized over $m=n-2$ sites. Therefore the square coupling of a given proton is divided by $\mathrm{m}^{2}$, while the number of protons which are connected with an electronic spin is multiplied by $m$. Taking into account the probability for different cluster sizes, eq. (4.7) becomes :

$\left.T_{1 \mathrm{PP}}^{-1}\right)_{\omega \rightarrow 0}=p \sum_{n=3}^{\infty} \frac{C(n)}{n-2}\left\langle a_{\mathrm{P}}^{2}\right\rangle(1+2 \xi) / 2 \Delta$

which gives numerically :

$$
\left.T_{1 \mathrm{PP}}^{-1}\right)_{\omega \rightarrow 0}=0.6 C\left\langle a_{\mathrm{P}}^{2}\right\rangle(1+2 \xi) / 2 \Delta .
$$

The NMP spin concentration becomes $C=16 \%$, $14 \%$ and $3 \%$ using spin density distributions $(a),(b)$ and $(c)$, respectively.

Table I displays the different values proposed so far for the back charge transfer in NMP TCNQ. Our results are consistent with the values obtained by the other methods, except the structural comparative study. However, a precise value cannot be obtained at the present time from nuclear relaxation experiments for lack of a reliable value for the hyperfine couplings in the NMP radical.

5. TCNQ spin dynamics. - Due to the smallness of the intrinsic TCNQ relaxation mechanism, we cannot neglect the influence of the NMP spins on the TCNQ protons. Therefore, in order to extract the intrinsic TCNQ rate $\left(T_{1 \mathrm{QQ}}^{-1}\right)$ we need to evaluate the contribution of the NMP spins (see eq. (3.1)) :

$$
T_{1 \mathrm{PQ}}^{-1}=C \frac{\pi}{10}\left[3\left\langle d_{\mathrm{PQ}}^{2}\right\rangle f_{\mathrm{P}}\left(\omega_{\mathrm{N}}\right)+7\left\langle d_{\mathrm{PQ}}^{2}\right\rangle f_{\mathrm{P}}\left(\omega_{\mathrm{e}}\right)\right]
$$


TABLE I

Back charge transer in NMP TCNQ

\begin{tabular}{|c|c|c|c|c|c|c|}
\hline Method & NMR & TEP & $\begin{array}{l}\text { Bond } \\
\text { length }\end{array}$ & X-ray & X-ray & $T_{1}$ \\
\hline - & - & - & - & - & - & - \\
\hline Reference & 9 & 10 & 11 & 12 & 26 & $\begin{array}{c}\text { Present work } \\
\left({ }^{3}\right)\end{array}$ \\
\hline$c$ & 0.06 & $0-0.2$ & 0.58 & $0.094\left(^{1}\right)$ & $0.181\left(^{2}\right)$ & $\begin{array}{l}\text { a) } 0.16 \\
\text { b) } 0.14 \\
\text { c) } 0.03\end{array}$ \\
\hline
\end{tabular}

(1) By assuming $4 k_{\mathrm{F}}$ scattering.

$\left({ }^{2}\right)$ From a different interpretation of data of reference 12.

$\left.\left.\left.{ }^{3}\right) a\right), b\right)$, and c) refer to spin density distributions $(a),(b)$ and (c) in figure 2.

The hyperfine cross coupling $d_{\mathrm{PO}}$ between NMP spins and TCNQ protons is purely dipolar. It is expressed as follows :

$$
\left\langle d_{\mathrm{PQ}}^{2}\right\rangle=\left(\hbar \gamma_{\mathrm{e}} \gamma_{\mathrm{N}}\right)^{2} \frac{1}{4} \sum_{\lambda=1}^{4} \sum_{q}\left(\sum_{i} \frac{\sigma_{i}}{r_{i q \lambda}^{3}}\right)^{2}
$$

where $r_{i q \lambda}$ is the distance between the proton $\lambda(\lambda=1$ to 4$)$ of a given TCNQ and the atom $i$ of the NMP radical $q$. Thus, $\left\langle d_{\mathrm{PQ}}^{2}\right\rangle$ can be calculated using the crystallographic structure of NMP TCNQ [24] and the different NMP spin density models. On the other hand, $C f_{\mathrm{P}}\left(\omega_{\mathrm{N}}\right)$ and $C f_{\mathrm{p}}\left(\omega_{\mathrm{e}}\right)$ are known from the NMP spin dynamics analysis. Therefore one obtains an evaluation of the NMP spin contribution to the TCNQ proton relaxation. It should be pointed out that this correction is dependent on the spin density model for the NMP molecule, but not on the possible delocalization of the NMP spin over several sites, because it involves the ratio $\left\langle d_{\mathrm{PQ}}^{2}\right\rangle \mid\left\langle a_{\mathrm{P}}^{2}\right\rangle$ which is nearly the same whatever the delocalization of the electronic NMP spin. One has also to take into account the incomplete deuteration of (NMP)*. In the measured value of $T_{1 \mathrm{Q}}\left(T_{1 \mathrm{Q}(\mathrm{m})}\right)$ there is an extra contribution coming from the remaining protons in the (NMP)* stacks. Since $16 \%$ of the deuterated phenazinium molecules have a remaining proton, one has :

$$
T_{1 \mathrm{Q}(m)}^{-1}=T_{1 \mathrm{Q}}^{-1}+0.04 T_{1 \mathrm{P}}^{-1} .
$$

Subtracting the extra contributions, the intrinsic TCNQ relaxation rate $T_{10 Q}^{-1}$ is finally obtained.

The resulting frequency dependence of $T_{100}^{-1}$ is given in figure 4. It is plotted versus the inverse square root of the nuclear Larmor frequency $\left(F=\omega_{\mathrm{N}} / 2 \pi\right)$ as is usual for a test of a 1D diffusive behaviour for the spin dynamics [27, 28]. It appears that the model used for the NMP spin density strongly affects the result. However, it turns out that, whatever the model used, one obtains a linear variation :

$$
T_{1 \mathrm{QQ}}^{-1}=A+B F^{-1 / 2}
$$

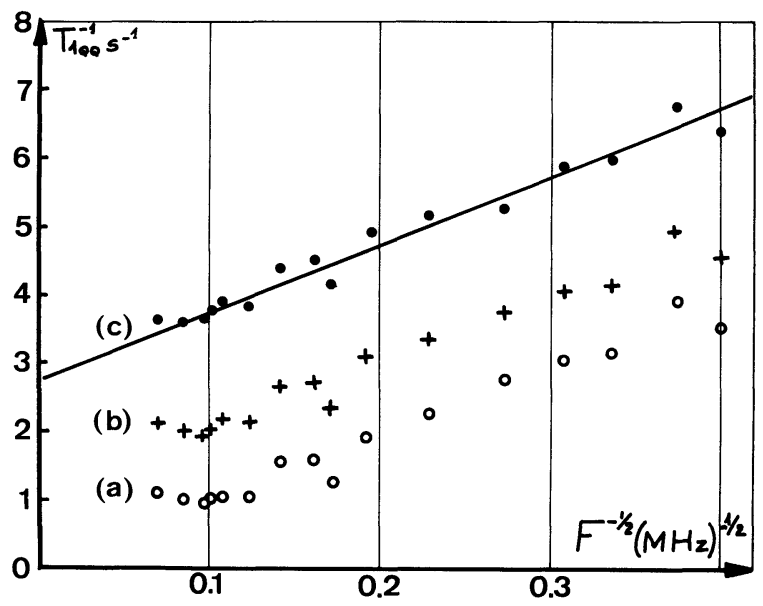

FIG. 4. - Intrinsic TCNQ proton relaxation rate as a function of the inverse square root of the nuclear Larmor frequency. $(a),(b)$ and $(c)$ refer to the spin density distributions of figure 2 .

which clearly establishes the 1D diffusive behaviour of the spin fluctuations in the TCNQ stacks. Furthermore the value of the slope $B$ is nearly model-independent, thus a reliable value can be deduced for the diffusion coefficient. $B$ is related to the diffusive part of the spin correlation function by the expression $[7,29]$ :

$$
\frac{B}{\sqrt{F}}=\frac{1}{2} a_{\mathrm{Q}}^{2} F_{\mathrm{Q}}\left(\omega_{\mathrm{e}}\right)=\frac{1}{2} a_{\mathrm{Q}}^{2} \frac{k T \tilde{\chi}_{\mathrm{Q}}}{\sqrt{2 \tilde{D} \omega_{\mathrm{e}}}} .
$$

In eq. (5.5) we have only retained the scalar part of the hyperfine coupling in the TCNQ molecule $a_{\mathrm{Q}}$. We have also supposed that the $1 \mathrm{D}$ diffusive motion is observed at $\omega_{\mathrm{e}}$ and not at $\omega_{\mathrm{N}}$ [28]. To estimate $\tilde{D}$ from eq. (5.5) we assume that :

i) the hyperfine coupling has the same value as that measured in solution : $a_{\mathrm{Q}} / \gamma_{\mathrm{e}}=1.5 \mathrm{G}$ [22].

ii) the NMP spin susceptibility follows a Curie law; thus : $\tilde{\chi}_{\mathrm{Q}}=\tilde{\chi}-C /(2 k T)$, where $\tilde{\chi}$ is obtained from the static spin susceptibility : $\tilde{\chi}=\chi /\left(2 N \mu_{\mathrm{B}}^{2}\right)$. We then obtain $: \tilde{D} \sim 6.10^{14} \mathrm{rad}$./s. This value is similar to 
those obtained in other well-conducting TCNQ salts. It turns out that there is no fundamental difference concerning the spin dynamics at room temperature in the TCNQ chain for the three conducting compounds : TTF TCNQ [7], QnTCNQ 2 [28] and NMP TCNQ. However, it appears that a cut-off effect has been observed in TTF TCNQ [7], while it is not observed for $\mathrm{QnTCNQ}_{2}$ and NMP TCNQ for nuclear frequencies as low as $6 \mathrm{MHz}$ [30].

The results of figure 4 have also been plotted on a logarithmic scale in figure 1 . It has been claimed by Butler et al. [31] that such a representation would give a better test for a 1D diffusive motion. However, taking into account the possibility of a constant term $A$, eq. (5.4) does not give a straight line with a slope $1 / 2$ in a log-log plot. A sizable value of $A$ could arise from both the $\omega_{\mathrm{N}}$ contribution (if the diffusion is observed at $\left.\omega_{\mathrm{e}}\right)[17,28]$ and from a non diffusive contribution at $\omega_{\mathrm{e}}$ [7].

6. Conclusion. - We have shown that the spin dynamics of TCNQ stacks has a 1D diffusive behaviour as in $\mathrm{QnTCNQ}_{2}$ and TTF TCNQ. No strongly enhanced dynamic susceptibility effect needs to be invoked to explain the moderate value of the intrinsic TCNQ relaxation rate. Moreover the present results rule out the interpretation previously proposed to explain the temperature variation of $T_{1}$ in NMP TCNQ [28, 32]. The TCNQ electronic spins provide only a small contribution to the nuclear relaxation. Most of the relaxation is due to the smaller number of electronic spins which are localized on the NMP stacks. A direct proof of the incomplete charge transfer in NMP TCNQ is thus given from nuclear relaxation. The amount of back charge transfer can be estimated, but a definite value cannot be extracted form $T_{1}$ data until a reliable model is available for the spin density distribution over the NMP radical. Anyway, using different sets of spin density data, it appears that the amount of back charge transfer is smaller than $20 \%$. This result is in qualitative agreement with other experimental determinations $[9,10,12]$, but not with structural considerations which give a larger value [11].

Acknowledgments. - We are greatly indebted to J. Douady, Y. Ellinger, Cl. Jeandey and R. Subra for computational help in the MO calculations.

\section{References}

[1] For recent reviews see : ShChegolev, I. F., Phys. Status Solidi (A) 12 (1972) 9.

André, J. J., Bieber, A. and Gauthier, F., Ann. Phys. 1 (1976) 145

[2] Burarov, L. I., Fedutin, D. N. and Schchegolev, I. F., Sov. Phys. JETP 32 (1971) 612.

[3] Epstein, A. J., Etemad, S., Garito, A. F. and Heeger, A. J., Phys. Rev. B 5 (1972) 952.

[4] Ehrenfreund, E., Rybaczewski, E. F., Garito, A. F. and Heeger, A. J., Phys. Rev. Lett. 28 (1972) 875.

[5] Ehrenfreund, E., Etemad, S., Cole an, L. B., Rybaczewski, E. F., Garito, A. F. and Heeger, A. J., Phys. Rev. Lett. 29 (1972) 269.

[6] Rybaczewski, E. F., Garito, A. F., Heeger, A. J. and EhrenFREUND, E., Phys. Rev. Lett. 34 (1975) 524.

[7] Soda, G., Jérome, D., Weger, M., Fabre, J. M. and Giral, L., Solid State Commun. 18 (1976) 1417.

Soda, G., Jérome, D., Weger, M., Alizon, J., Gallice, J., Robert, H., Fabre, M. and Giral, L., J. Physique 38 (1977) 931.

[8] Torrance, F. B., Scott, B. A. and Kaufman, F. B., Solid State Commun. 17 (1975) 1369.

[9] Butler, M. A., Wudl, F. and Soos, Z. G., Phys. Rev. B 12 (1975) 4708

[10] Kwak, J. F., Beni, G. and Chaikin, P. M., Phys. Rev. B 13 (1976) 641.

[11] Flandrois, S. and Chasseau, D., Acta. Crystallog. B 33 (1977) 2744.

[12] Ukei, K. and Shirotani, I., Commun. Phys. 2 (1977) 159.

[13] Butler, M. A., Wudl, F. and Soos, Z. G., J. Phys. Chem. Solids 37 (1976) 811.

[14] Melby, L. R., Can. J. Chem. 43 (1965) 1448.

[15] Vivian, D. L., J. Org. Chem. 21 (1956) 822.

FisCHER, G., Synthesis 4 (1973) 218.
[16] Ehrenfreund, E. and Garito, A. F., Solid State Commun. 19 (1976) 815

[17] Devreux, F. and Nechtschein, M., Solid State Commun. 16 (1975) 275.

Travers, J. P., unpublished work.

[18] Devreux, F., Boucher, J. P. and Nechtschein, M., J. Physique 35 (1974) 271.

[19] Avalos, J., thèse de $3^{e}$ cycle, Grenoble (1977) unpublished;

Avalos, J., Devreux, F., Guglielmi, M. and NechtsCHEIN, M., Molecular Physics, to be published.

[20] Berlinsky, A. J., Carolan, J. F. and Weiler, L., Solid State Commun. 15 (1974) 795.

[21] Hennessy, M. J., McElwee, C. D. and Richards, P. M., Phys. Rev. B 7 (1973) 930.

[22] Fischer, P. H. and MaC Dowell, C. A., J. Am. Chem. Soc. 85 (1963) 2694.

[23] Hausser, K. H., Habich, A. und Franzen, V., Z. Naturforsch A 16 (1961) 836.

[24] Fritchie, C. J., Acta Crystallogr. 20 (1965) 892.

[25] Holczer, K., Mihaly, G., Janossy, Á., Grüner, G. and KERTESZ, M. preprint.

[26] Kondo, J. and Yamaji, K., J. Phys. Soc. Jpn 43 (1977) 424.

[27] Borsa, F. and Mali, M., Phys. Rev. B 9 (1974) 2215.

Ahmed-Bakheit, M., BarjhouX, Y., Ferrieu, F., NechtsCHeIN, M. and Boucher, J. P., Solid State Commun. 15 (1974) 25.

[28] Devreux, F., Phys. Rev. B 13 (1976) 4651.

[29] Devreux, F., Bulletin du DRF 11 (1977) 86.

[30] A detailed comparison of the spin dynamics properties in these three compounds will be published later on.

[31] Butler, M. A., Walker, L. R. and SooQ, Z. G., J. Chem. Phys. 64 (1967) 3592.

[32] Hone, D. and Pincus, P., Phys. Rev. B 7 (1973) 4889. 